\title{
EDITORIAL
}

\section{Problem shooting in obstetrics}

\author{
Prof (Dr) Hem Kanta Sarma
}

\author{
Correspondence: Prof (Dr) Hem Kanta Sarma, Professor \& HOD, Department of Obstetrics \\ \& Gynaecology, Jorhat Medical College, Jorhat, Assam, India; \\ Email - sarmahemkanta@gmail.com
}

Distributed under Attribution-Non Commercial-Share Alike 4.0 International (CC BY-NC-SA 4.0)

An obstetrician is always at risk of various unanticipated problems. Even a simple normal delivery may end up with severe postpartum haemorrhage $(\mathrm{PPH})$ which might be leading to maternal death. Simple episiotomy may lead to catastrophic haematoma. To face this type of challenges the obstetrician should always follow certain basic principles of surgery and medicine. Hematoma or bleeding in episiotomy occurs in $18 \%$ cases ${ }^{1} .0 .8 \%$ cases delivered vaginally suffers from severe $\mathrm{PPH}{ }^{2}$. But there are certain exceptional situations where presentation is not as usual and it misleads even a veteran obstetrician. Uterocutaneous fistula following uterine surgery, specially caesarean section is a rare condition ${ }^{3}$. But there are instances as mentioned in this article in case no - 3. To manage a few cases of secondary $\mathrm{PPH}$, bilateral internal iliac ligation has to be performed. Bilateral internal iliac ligation reduces the pelvic arterial blood flow by $49 \%$ and pelvic pressure by $85 \%{ }^{4}$, thus facilitates the maternal system of haemostasis to stabilize.

In this article I would like to throw some light in this aspect by sighting some real case scenarios faced by the author himself while playing the role of problem shooter.

\section{Case 1}

Primigravida 32 years, term pregnancy, delivered spontaneously vaginally following right medio-lateral episiotomy. Patient complained of bleeding per vaginum. On examination small oozing was noticed from the wound. In operation theatre, under anesthesia wound was explored. Few mattress sutures were given overlapping the wound area. Bleeding stopped. After 3 days again fresh bleeding was noticed. Re-exploration under general anesthesia was done and stitches applied. Blood transfused. Broad spectrum antibiotic was given. Again after 2 days fresh bleeding was noticed from the wound.
Remedy: Patient was taken to operation theatre. Under anesthesia the wound was completely opened removing all the old stitches. Washing of the tissue was done thoroughly. Waited for few minutes and observed carefully for the bleeding points after soaking gently the oozing blood. Exact bleeding points were identified, then tied and then the wound was repaired in layers as usual.

\section{Case 2}

A second gravida 30 years old woman had uneventful caesarean section in a peripheral hospital and started bleeding per vaginum torrentially after 1 week of delivery at home. Patient was re-admitted. Uterine cavity was explored for retained product. During this process, there was perforation of uterus. Laparotomy was performed. Hysterectomy was attempted. But hysterectomy had to stop after cutting and tying the broad ligaments due to severe bleeding. Abdomen was closed and referred to a tertiary hospital. The patient was treated initially in the tertiary hospital conservatively by antibiotics, blood transfusion and prostaglandins but bleeding continued.

Remedy: Bilateral internal iliac artery ligation was done by extraperitoneal route as the intra abdominally tissues were fragile and infected. Patient recovered completely. After 2 weeks of internal iliac ligation there was sudden bout of vaginal bleeding. This time a total abdominal hysterectomy was performed without any difficulty. She recovered well. Subsequent follow up of the patient was fine.

\section{Case 3}

Primigravida, 28 years old woman had elective caesarean section in a private hospital for cephalo-pelvic disproportion (CPD). Patient was re-admitted after 10 days of operation with complaints of fever, pain at wound and vaginal bleeding. Initially she was treated conservatively. Gradually 
The New Indian Journal of OBGYN. 2021 (July-December); 8(1)

the wound became wet with copious, purulent and bloody discharge. General condition of the patient deteriorated. Patient was referred to a tertiary care hospital where after all the investigations it was found that there was wound dehiscence with a fistulous track from the uterine wound with the abdominal wound. Patient was treated conservatively with blood transfusion, protein and vitamin supplementation, culture guided broad spectrum antibiotics and local dressing. After improvement of general condition of the patient, laparotomy was performed.

While fistulous track was excised with excision of the margins of the uterine scar followed by repair of uterus and abdominal wound was done. Peritoneal toileting was done. Drains kept in the peritoneal cavity and abdominal wound. There was uneventful complete recovery.

Comment: It was informed that in this patient during caesarean section the wound was closed in single layer. There was subsequent hematoma formation on the caesarean section wound which got infected in due course leading to secondary PPH. Similarly there was wound hematoma on the abdominal wall wound which leads to abscess formation, eventually leading to fistulous communication in between the two wounds.

\section{Conclusion}

While managing a patient of post operative bleeding - the wound is to be explored thoroughly after removing all the stiches to visualise the bleeding point. A blind stitch is unlikely to help, rather harms due to tissue oedema and inflammation.

In an infected case, it is always better to avoid tissue handling of the friable area and choose an alternative pathway such as extraperitoneal route for internal iliac ligation.

Internal iliac ligation is helpful in managing intractable secondary PPH. But after establishment of collateral circulation sometimes there might be recurrence of vaginal bleeding where a hysterectomy may be a helpful option.

Newer technique of surgery like single layer closure of caesarean section is not a panacea. Only in selected cases and selected situations these can be exercised otherwise it may lead to catastrophic complications.

\section{Conflict of interest: None. Disclaimer: Nil.}

\section{References}

1. Suleimanzadeh Mousavi SH, Miri M, Farzaneh F. Episiotomy and its complications. Zaheedan J Res Med Sci. 2020; 23(2): e104127.

2. Horeyda F, Mackenzie IZ. Secondary post partum haemorrhage: Evidence, mortality and current management. BJOG: Am International J. Obstet \& Gynaecology. 2001; 108(9): 927-30.

3. Rezaci Z, Shahraki Z, Shirazi M. Uterocutaneous fistula as a rare complication after caesarean delivery; case series. J Obstet Gynaecol cancer Res. 2017; 2(3): e14071

4. Seleuk I, Uzuner B, Boduc E, Baykus Y, Akar B, Gungor T. Step by step ligation of the internal iliac artery. J Turk Ger Gynecol Assoc. 2019 Jan; 20(2): 123-28. 